\title{
Tuberculosis of the Oral Cavity and Associated Structures: The PGIMER Experience
}

\section{Vidya Rattan, Sachin Rai}

\begin{abstract}
Extrapulmonary tuberculosis of the oral cavity and its associated structures are diagnostic challenge. It is often misdiagnosed by the attending dentist who is the first among clinicians to come across such pathological entity. Lesions are often slow growing and painless and hence are initially neglected. Tubercular osteomyelitis of the jaw bone is a common occurrence in developing countries like India and often these cases first appear in dental out-patient clinic. The purpose of this paper is to highlight the involvement of oral and maxillofacial structures with few case reports and their concurrent diagnostic procedures.
\end{abstract}

Keywords: Tuberculosis, Osteomyelitis, Oral cavity.

How to cite this article: Rattan V, Rai S. Tuberculosis of the Oral Cavity and Associated Structures: The PGIMER Experience. J Postgrad Med Edu Res 2013;47(4):214-217.

Source of support: Nil

Conflict of interest: None declared

\section{INTRODUCTION}

Tuberculosis (TB) in oral cavity and its associated structures are rare manifestation of extrapulmonary tuberculosis (EPTB), occurring in approximately $3 \%$ of all cases. ${ }^{1}$ The diagnostic challenge due to its non-specific presentation may mislead the dentist or the clinician and delay the diagnosis. The purpose of this paper is to highlight the variable appearance of EPTB in oral cavity and its associated structures. We also present a pictorial series of few cases involving the soft and the hard tissue of the oral and maxillofacial region which were diagnosed, treated and followed in our unit.

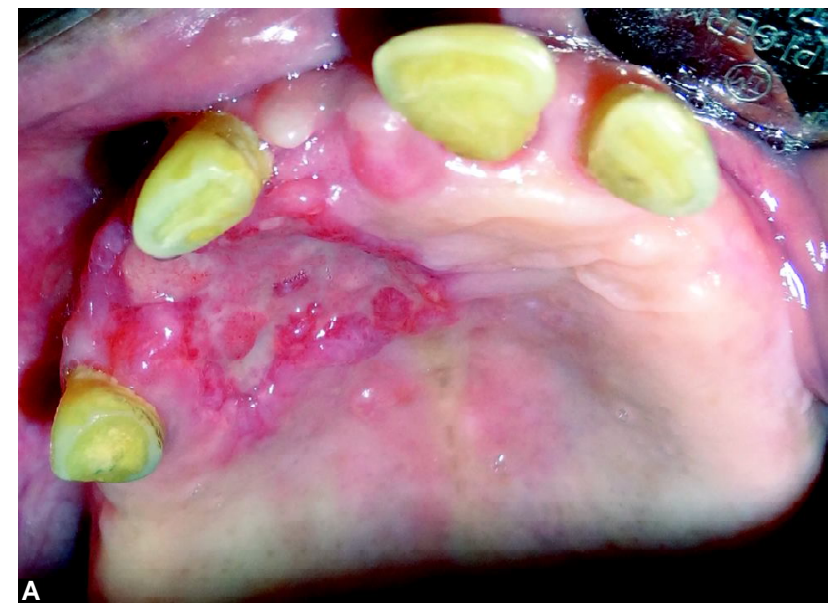

\section{LESIONS OF ORAL CAVITY AND ASSOCIATED SOFT TISSUE STRUCTURES}

Common sites for oral tubercular lesions occur are the tongue, palate, gingiva, floor of mouth, soft palate and mucobuccal folds. ${ }^{2}$ Tongue is the most common site of involvement and accounts for nearly half of the cases. ${ }^{3}$ The clinical presentation in oral cavity may be that of swelling, ulcer or irregular growth which may be single or multiple; ulcerated or non-ulcerated giving a false suspicion of malignancy (Figs 1A and B). TB of the salivary glands is usually secondary to infection of the oral cavity or primary pulmonary tuberculosis. Parotid gland is most commonly involved $^{4}$ (Figs $2 \mathrm{~A}$ and B). Clinical presentation can be acute or chronic. Acute presentation may resemble acute sialadenitis and clinical differentiation may be difficult. Cervical tubercular lymphadenitis may present as multiple matted firm swelling in the neck with a stud collar appearance.

An incisional biopsy or FNAC of the lesion for histopathological examination and microbial culture is mandatory to establish the diagnosis. At times, the histopathological findings may be suggestive of only granulomatous infection. This encompasses a wide range of pathologies rather than definitive TB. Therefore, the clinicians must rely upon the clinical and radiological appearance as evidence to diagnose EPTB.

\section{LESIONS OF THE JAW BONES}

The clinical presentation of a patient with jaw involvement with TB may range from painless jaw swelling (lumpy jaw)

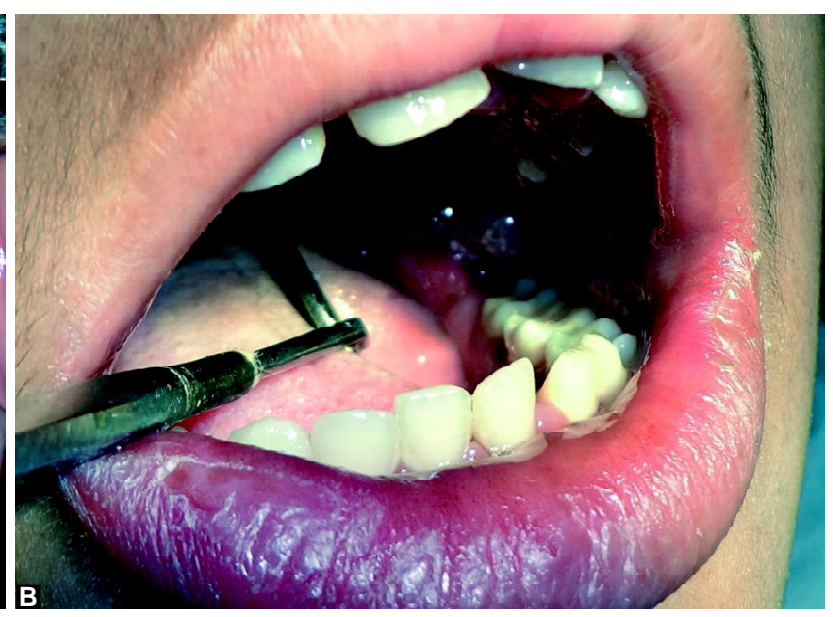

Figs 1A and B: Varied appearance of intraoral tubercular lesions 

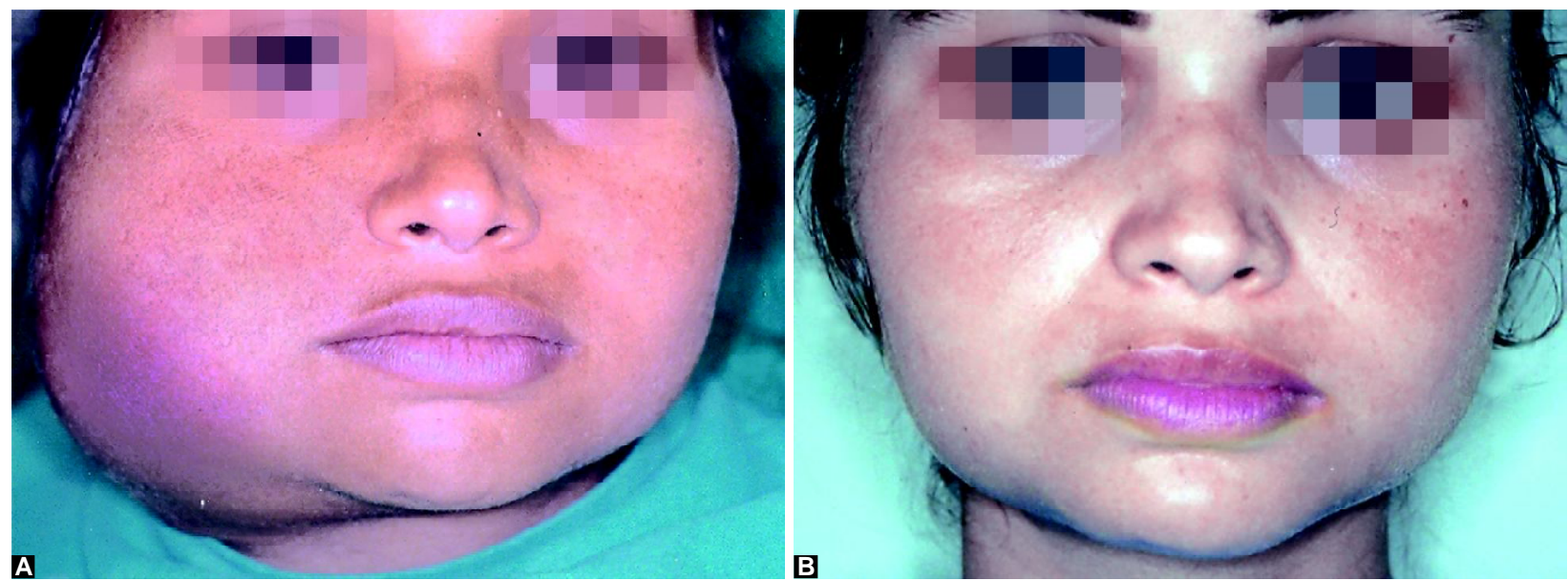

Figs 2A and B: Tubercular infection of the parotid gland before and after treatment

to draining sinus or osteomyelitis. ${ }^{5}$ Tubercular osteomyelitis of the mandible causes slow necrosis of the bone and may involve the entire mandible. The first demonstrable change is a small translucent area due to decalcification and blurring of trabecular details with irregular areas of radiolucency. The lesion is clinically undetectable at this stage. There is erosion of the cortex with little tendency to repair. Gradually, the bone is replaced by soft tuberculous granulation tissue. Caseation appears at places followed by softening and liquefaction. A subperiosteal abscess (lumpy jaw) then forms presenting as a painless, soft swelling (Figs 3A and B).

\section{LESIONS OF THE TEMPOROMANDIBULAR JOINT}

Primary TB of the Temporomandibular Joint (TMJ) is rare. Most of these patients present with preauricular swelling and trismus (Figs 4A and B). The clinical and radiological appearance of TB of the TMJ is non-specific and may be similar to that of arthritis, osteomyelitis or chronic joint diseases. The most common symptom is a painful preauricular swelling (unresponsive to antibiotics), associated with trismus. Radiographs and computed tomography (CT) scans show destruction of parts of the condyle and soft tissue masses. Radiological imaging, biopsy and culture usually confirm the diagnosis of a TB infection. ${ }^{5}$

\section{DISCUSSION}

EPTB is an uncommon form of chronic infection which does not present with the typical signs and symptom of pulmonary tuberculosis. Its diagnosis is often overlooked because it has no specific pathognomonic signs. It usually effects organs with suboptimal conditions for bacillary growth and hence has an insidious presentation and a slow evolution. These infections generally involve the head and neck through hematogenous or lymphatic routes. Orofacial tuberculosis is an uncommon form and presents at different sites, such as gingival, tongue, faucial pillars, muscles of mastication and buccal mucosa. EPTB also manifests as tubercular osteomyelitis of the jaw and TMJ. ${ }^{6}$

In a recent classification proposed by Andrade et al, the orofacial tubercular lesion has been subclassified into five
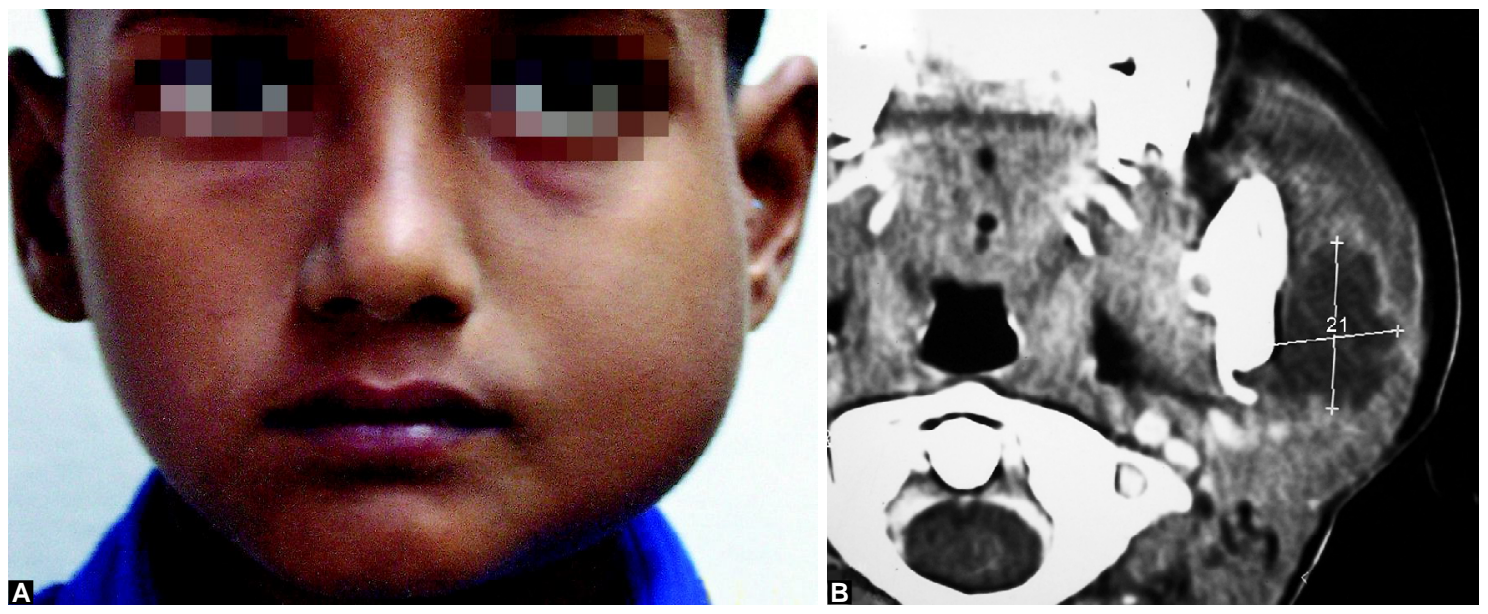

Figs $3 \mathbf{A}$ and $\mathbf{B}$ : Tubercular osteomyelitis of the ramus and angle involving the submasseter space of the mandible 

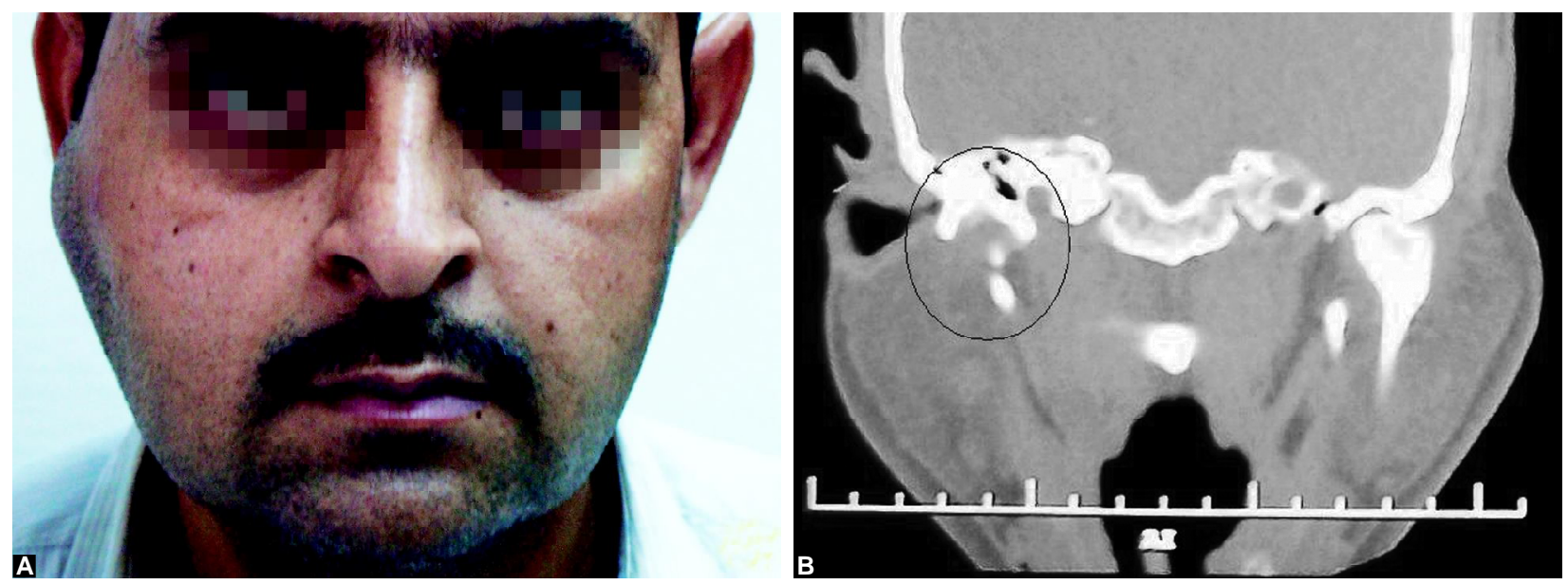

Figs 4A and B: Tubercular osteomyelitis of the condyle of the mandible

types. $^{7}$ Type I consists of lumpy jaw where the patient presents with an extraoral swelling without any intraoral or extraoral draining sinuses. Type II is where the patient reports with a history of extraction and present with a nonhealing extraction sockets with/without intraoral or extraoral draining sinus. In type III, the patient has no history of extraction and present with an intraoral or extraoral draining sinus in the orofacial region and an osteomyelitic bony lesion. Type IV has tuberculous lymphadenitis of the head, face and neck region without any features of types I, II, III or V, and type V lesions are those of sites in and around the oral cavity, e.g. maxillary antrum, salivary glands, orofacial muscles gingiva, tongue, etc.

Infection in the oral cavity is usually acquired through infected sputum coughed out by a patient with open pulmonary tuberculosis or by hematogenous spread. The integrity of the oral epithelium and the inhibitory effect of saliva are considered the reasons for the relative resistance to infection by bacilli. However, any break in the continuity of the oral epithelium owing to an abrasion, ulceration or an extraction socket facilitates inoculation of the bacilli present in the sputum, which is brought into intimate contact during coughing or speech. Predisposing local and systemic factors exist for the occurrence of oral lesions. These may be poor oral hygiene, local trauma, preexisting lesions, such as leukoplakia, periapical granuloma, cyst, abscess and periodontitis. A systemic predisposing factor, such as altered host resistance from immunosuppression or nutritional deficiencies, increases the susceptibility of a patient to a tubercular infection. ${ }^{8}$

Tuberculosis of jaw bone is an uncommon form of chronic osteomyelitis, occurring more often in young individuals and usually in late stages of the disease. Tuberculous osteomyelitis of jaw is very rare and constitutes less than $2 \%$ of the skeletal TB. Involvement can occur by hematogenous spread because of which the ramus of the mandible and the attached musculature are more affected. The lesion is seen to involve the outer cortical plates, whereas the medullary bone is less affected. Spread through blood vessels supplying the medial pterygoid and masseter muscles is considered the most important in the involvement of the mandibular ramus and body. ${ }^{9}$ Radiologically, tubercular osteomyelitis resembles non-specific osteomyelitis. Differential diagnosis includes actinomycosis lesion and other fungal infections. It has been suggested that MRI assessment improves the clinician's ability to make a differential diagnosis. MRI features of a TB infection in the TMJ may include synovitis, effusion, bone resorption and the presence of an abscess. ${ }^{10}$ If the condition is misdiagnosed as a common TMJ arthralgia or osteoarthritis, the joint destruction continues. The functional prognosis of the joint depends on the degree of destruction at the time of the correct diagnosis and start of treatment.

Tuberculous osteomyelitis of midfacial bones is extremely rare. Because of its rarity and variable presentation, this condition is difficult to diagnose, unless a high index of suspicion is kept in mind, which is more likely in an endemic area. Tuberculous osteomyelitis of skull bones accounts for 0.1 to $3.7 \%$ of all cases of skeletal tuberculosis. ${ }^{11}$ Three quarters of such cases are seen in patients less than 20 years of age, and half in those less than 10 years. The condition is usually secondary to a primary focus elsewhere, mainly in the lungs. Direct spread of infection from neighboring structures, such as the orbit, paranasal sinuses, face and nasal mucosa has also been implicated. ${ }^{12}$

\section{CONCLUSION}

In a tuberculosis-prevalent country, such as India, it is important to be aware of tubercular lesions involving the orofacial region. A long standing chronic lesion like ulcer, swelling or draining sinus in or around the oral cavity should raise suspicion of TB in the clinician's mind. Clinical 
presentation of EPTB is atypical. Especially when the disease involves obscure occult sites, EPTB may not even be considered in the initial list of differential diagnosis. Incisional biopsy for easily accessible sites and FNAC for deep-seated lesion can help the diagnosis. A histopathological examination and microbial culture should be promptly undertaken. Similarly, TB maybe suspected in situations where a routine therapy fails to bring about an improvement in the lesions. If diagnosed early, chemotherapy will cure the lesion and mutilating surgery can be avoided.

\section{REFERENCES}

1. Rivera H, Correa MF, Castillo-Castillo S, Nikitakis NG. Primary oral tuberculosis: a report of a case diagnosed by polymerase chain reaction. Oral Dis 2003 Jan;9(1):46-48.

2. Pasticci MB, Floridi P, Schiaroli E, Stagni GM, De Socio GV, Longari F, Baldelli F. Lingual tuberculosis: a rare disease in Western countries. New Microbiol 2012 Apr;35(2):233-237.

3. Kannan S, Thakkar P, Dcruz AK. Tuberculosis masquerading as oral malignancy. Indian J Med Paediatr Oncol 2011 Jul;32(3): 180-182.

4. Tanwar R, Iyengar AR, Nagesh KS, Jhamb P. Primary tuberculosis: an unusual finding in the oral cavity. Oral Health Dent Manag 2012 Mar;11(1):23-28.

5. Kamala R, Sinha A, Srivastava A, Srivastava S. Primary tuberculosis of the oral cavity. Indian J Dent Res 2011 Nov-Dec; 22(6):835-838.

6. Prasad PM. Primary tuberculosis of tonsils: a case report. Case Report Med 2012;2012:120382.
7. Andrade NN, Mhatre TS. Orofacial tuberculosis: a 16-year experience with 46 cases. J Oral Maxillofac Surg 2012;70: e12-e22.

8. Peck MT, Stephen LX, Marnewick J, Majeed A. Palatal ulceration as the first sign of pulmonary tuberculosis: a case report. Trop Doct 2012 Jan;42(1):52-53.

9. Soman D, Davies S. A suspected case of tuberculosis of the temporomandibular joint. J Br Dent J 2003 Jan 11;194(1):23-24.

10. Sheikh S, Pallagatti S, Gupta D, Mittal A. Tuberculous osteomyelitis of mandibular condyle: a diagnostic dilemma. Dentomaxillofac Radiol 2012 Feb;41(2):169-174.

11. Mignogna MD, Muzio LL, Favia G, Ruoppo E, Sammartino G, Zarrelli C, Bucci E. Oral tuberculosis: a clinical evaluation of 42 cases. Oral Dis 2000 Jan;6(1):25-30.

12. Baruah B, Goyal A, Shunyu NB, Lynrah ZA, Raphael V. Tuberculosis of nose and palate with vanishing uvula. Indian $\mathbf{J}$ Med Microbiol 2011 Jan-Mar;29(1):63-65.

\section{ABOUT THE AUTHORS}

\section{Vidya Rattan}

Additional Professor, Unit of Oral and Maxillofacial Surgery Department of Oral Health Sciences, Postgraduate Institute of Medical Education and Research, Chandigarh, India

\section{Sachin Rai (Corresponding Author)}

Assistant Professor, Unit of Oral and Maxillofacial Surgery Oral Health Sciences Centre, Postgraduate Institute of Medical Education and Research, Chandigarh, India, Phone: 9872987660 e-mail: drraisachin@gmail.com 